\title{
A randomised controlled trial to investigate the use of high-frequency airway oscillations as training to improve dyspnoea in COPD
}

\author{
Enya Daynes $\mathbb{1}^{1,2,3}$, Neil Greening ${ }^{1,2,3}$, Salman Siddiqui ${ }^{2,3}$ and Sally Singh ${ }^{1,2,3}$
}

Affiliations: ${ }^{1}$ Centre of Exercise and Rehabilitation Sciences, Glenfield Hospital, Leicester, UK. ${ }^{2} \mathrm{NIHR}$ Leicester Biomedical Research Centre - Respiratory, Glenfield Hospital, Leicester, UK. ${ }^{3}$ University of Leicester, Leicester, UK.

Correspondence: Enya Daynes, Centre of Exercise and Rehabilitation Sciences, NIHR Leicester Biomedical Research Centre - Respiratory, Glenfield Hospital, Groby Road, Leicester, LE3 9QP, UK.

E-mail: enya.daynesQuhl-tr.nhs.uk

\section{ABSTRACT}

Introduction: Chronic obstructive pulmonary disease (COPD) is characterised by expiratory flow limitation resulting in symptomatic dyspnoea, sputum retention and ventilation heterogeneity. Changes in breathing mechanics affect the ability of respiratory muscles to respond to the ventilatory demands, increasing the sensation of dyspnoea. A high-frequency airway oscillating device has been developed to combine respiratory muscle training and oscillations to improve dyspnoea and sputum retention within COPD.

Methods and analysis: Patients with symptomatic COPD (Medical Research Council Breathlessness scale grade $\geqslant 2$ ) will be recruited to a double-blind, randomised, sham-controlled trial. Both groups will follow an 8 -week intervention phase using the device three times per day for $5 \mathrm{~min}$ at a time. This will be recorded in a self-reported diary. The device applies a flow resistive load and oscillations for combined training. Those receiving the sham device will follow the same protocol; however, the mechanism of action will be removed from the device. Improvements in the Chronic Respiratory Questionnaire-Dyspnoea domain will be the primary outcome measure. Secondary outcomes will explore respiratory muscle function, health-related quality of life, exercise capacity and physical activity. The Lung Clearance Index will be an exploratory outcome. Outcomes will be explored using the most appropriate statistical test, dependent on the sample distribution. Focus groups will be an exploratory outcome and analysed by thematic analysis.

Ethics and dissemination: Ethical approval has been obtained from the East Midlands-Leicester South Research Ethics Committee and the trial has been registered through the ISRCTN Registry. The study results will be disseminated to the appropriate stakeholders through presentations, conferences and peer-reviewed journals.

@ERSpublications

A double-blind, placebo-controlled, randomised trial investigating the effects of high-frequency airway oscillations on dyspnoea, sputum, quality of life and exercise capacity in \#COPD. http://bit.ly/2F8bQ6s

Cite this article as: Daynes E, Greening N, Siddiqui S, et al. A randomised controlled trial to investigate the use of high-frequency airway oscillations as training to improve dyspnoea in COPD. ERJ Open Res 2019; 5: 00064-2019 [https://doi.org/10.1183/23120541.00064-2019].

This study is registered at www.isrctn.com with identifier number ISRCTN45695543. All individual participant data collected during the trial after de-identification will be available on request by researchers who provide a methodologically sound protocol.

Received: March 082019 | Accepted after revision: June 042019

Copyright $\odot$ ERS 2019. This article is open access and distributed under the terms of the Creative Commons Attribution Non-Commercial Licence 4.0. 


\section{Introduction}

Chronic obstructive pulmonary disease (COPD) is characterised by expiratory flow limitation that results in excessive dyspnoea, reduced exercise tolerance and reduced health-related quality of life. Dyspnoea is a multidimensional symptom with many mechanisms contributing to the perception of dyspnoea. It is known that respiratory muscle dysfunction can contribute to dyspnoea and that this may be a result of the inability of respiratory muscles to meet the demands of the mechanical load and capacity, leading to respiratory muscle dysfunction and an increased sensation of dyspnoea [1]. Persistent and recurrent dyspnoea impacts activity levels and the ability of patients to perform activities of daily living, which impacts quality of life. Respiratory muscle training can be used to address this mechanism of dyspnoea. Research primarily explores the use of inspiratory muscle training in COPD, demonstrating improvements in inspiratory muscle strength and endurance, functional exercise capacity, and health-related quality of life when compared with a control group [2]. These improvements appear meaningful in terms of quality of life, dyspnoea and muscle strength [3].

Combined inspiratory and expiratory respiratory muscle training is an additional technique for the management of dyspnoea; however, this technique is used much less frequently. This may be due to the opinion that patients with COPD do not demonstrate expiratory muscle weakness, although it has been suggested that up to $50 \%$ of patients with moderate to severe COPD exhibit expiratory muscle weakness in parallel with inspiratory muscle weakness [4]. Inspiratory muscle weakness is defined by a maximal inspiratory pressure generating capacity of $\leqslant 60 \mathrm{cmH}_{2} \mathrm{O}$. This value has some limitations in that it is not adjusted for age, height and weight. The values for expiratory muscle weakness are not definitive. EvANs and WHITELAW [5] present a calculation that may be useful in identifying patients who demonstrate expiratory muscle weakness; however, lack of consensus on expiratory muscle weakness definitions limits targeted therapy [6]. Percentages of predicted values can be calculated for both inspiratory and expiratory muscle strength based of the Evans and Whitelaw [5] formula. There is a small evidence base for combined respiratory muscle training; however, it has been demonstrated to improve respiratory muscle strength and endurance, with an increase in 6-min walk distance when compared with inspiratory muscle training alone [7]. The mechanism and effectiveness of combined respiratory muscle training are not fully accepted as a treatment strategy for patients with COPD. The Joint American College of Chest Physicians/American Association for Cardiovascular and Pulmonary Rehabilitation Committee declared that a stimulus or load applied to the respiratory muscles during training is sufficient to augment respiratory muscle strength training and is associated with increased exercise capacity and decreased dyspnoea [8]. However, this is not included in the National Institute for Clinical Excellence guidelines due to disparity in the research $[6,8]$.

Sputum retention is commonly reported alongside dyspnoea as an unsettling symptom for patients with COPD. Additional therapy is available for the management of sputum retention, and can include manual therapies, breathing exercises and devices as an adjunct [9]. Management of sputum has been demonstrated to reduce hospital admissions, need for ventilatory assistance and quality of life through various methods of sputum clearance, including manual therapies, breathing exercises and positive expiratory pressure devices in patients with COPD [10]. The use of devices has a smaller evidence base compared with other techniques. This is even more limited within COPD management; however, oscillatory devices aim to break down the enzymes in the sputum and vibrate the secretions of the chest wall, making them easier to clear. There is a disparity in the current literature base due to the inability to reliably capture sputum clearance. Sputum wet and dry weight measurements are labour intensive and difficult to translate into meaningful results. Patient-reported outcome measures may provide some insight into the impact of sputum, but this can be variable. Therefore, we propose the use of the Lung Clearance Index (LCI) as a surrogate measure of sputum clearance.

The LCI has been used in cystic fibrosis, paediatrics and early lung disease, and has been identified as more sensitive than conventional spirometry and is responsive to inhaled treatment [11]. The LCI is calculated via a multiple breath washout system using an inert gas and is determined by the cumulative exhaled volume divided by the functional residual capacity. This technique gives insight into the unevenness of ventilation due to pathological changes in lung structure. Ventilation heterogeneity assesses the gas mixing properties of the lungs, which are known to be affected in COPD. Other measures of the multiple breath washout provide further insight into large and small airways disease. Sputum plugging can occur at different anatomical levels within both the large and small airways, and therefore phase 3 slope analysis gives insight into the ventilation heterogeneity in the conducting ( $S$ cond) and acinar ( acin) airways, respectively. It is plausible that an improvement in sputum clearance could impact the gas mixing properties and therefore impact on the LCI. The LCI is repeatable over short and long time periods (up to 8 weeks) in a cohort of COPD patients; however, its response to therapy is yet to be explored [12-14].

The Aerosure Medic is a dual-function device for dyspnoea management and secretion clearance. It is a high-frequency airway oscillation (HFAO) device that offers flow resistance to inspiration and expiration in order to provide respiratory muscle training with the aim to reduce dyspnoea and improve breathing 
efficiency [15]. The device also offers oscillations for mucociliary clearance on inspiration and expiration that may contribute to improved quality of life and reduction of dyspnoea by addressing airflow obstruction [15]. This device is commercially available for patients; however, there are limited data on its clinical effectiveness. A feasibility study was conducted prior to the proposed randomised controlled trial in order to further understand the mechanism of action and effect on symptoms, quality of life and study procedures [15]. The study recruited 24 participants, and the results demonstrated its feasibility with high recruitment rate, compliance and low attrition. Trends were noted in improving dyspnoea, respiratory muscle strength and health-related quality of life. The trial protocol was deemed appropriate and the training intervention was manageable, and therefore the results of the feasibility study encouraged a clinical effectiveness trial.

The objectives for the clinical effectiveness trial are: 1) to determine the effects of a HFAO device on dyspnoea, lung function, health-related quality of life, exercise capacity and physical activity in participants with COPD when compared with a sham device; 2) to explore if there is a subgroup of patients who receive the most benefit from this therapy; 3) to explore the LCI and other measures of multiple breath washout as a measure of sputum clearance in participants with COPD and its response to an intervention; 4) to explore the impact of oscillatory positive expiratory pressure on the LCI; and 5) to explore patients' experience and beliefs of using a device for the management of COPD.

\section{Methods and analysis}

This is a double-blind, randomised controlled trial comparing a HFAO device with a sham device. The trial is registered with the ISRCTN Registry (identifier ISRCTN45695543) and has been adopted onto the UK National Institute for Health Research (NIHR) Clinical Research Network portfolio.

A flow diagram of the study procedures is shown in figure 1 .

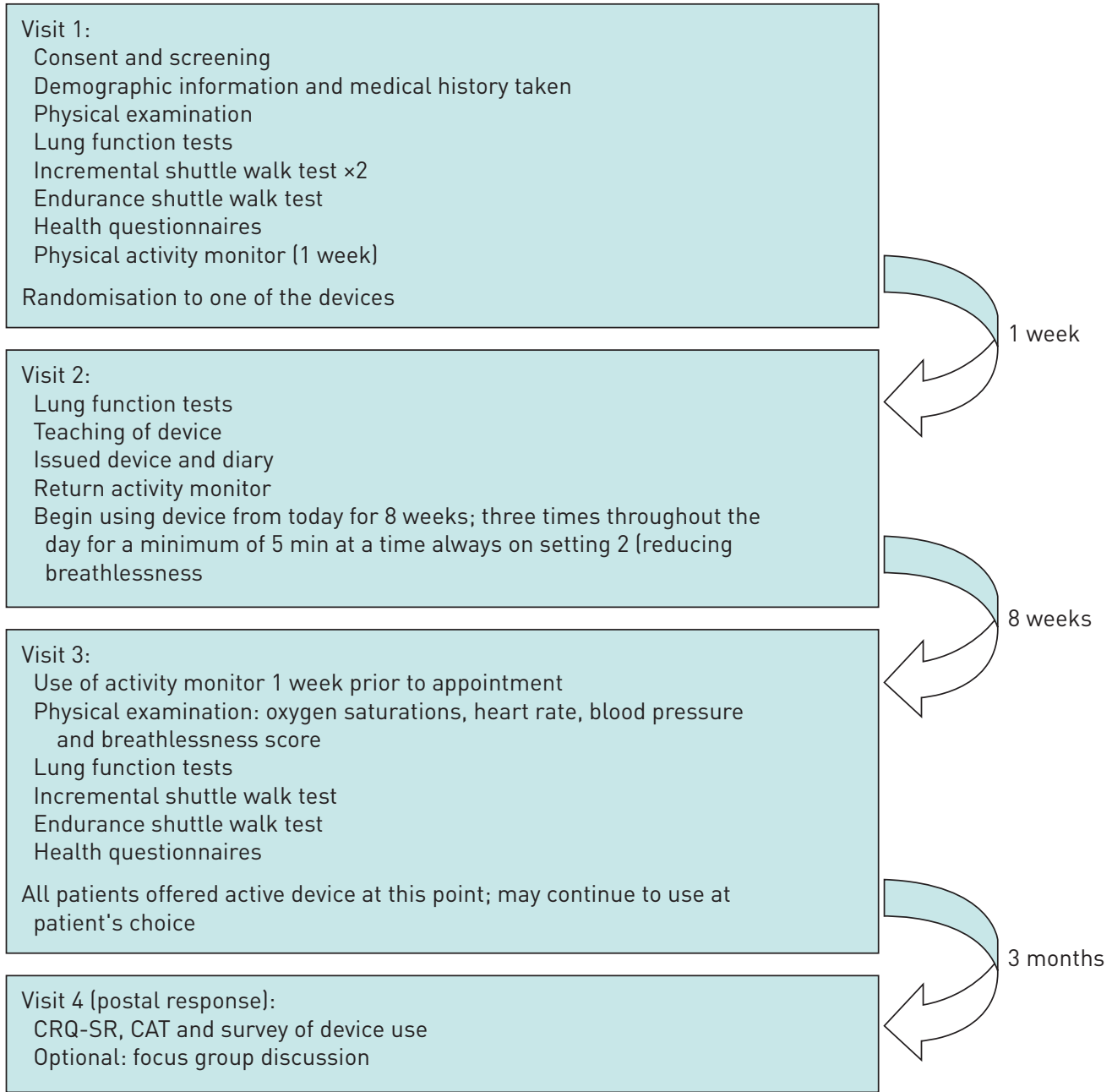

FIGURE 1 Flow diagram of study procedures in patients with symptomatic chronic obstructive pulmonary disease (COPD). CRQ-SR: self-reported Chronic Respiratory Questionnaire; CAT: COPD Assessment Test. 


\section{Participants}

Participants are considered eligible if they have an established diagnosis of COPD, confirmed by spirometry as defined by a forced expiratory volume in 1 s/forced vital capacity ratio $<0.7[16]$. Participants must also report significant dyspnoea identified on the Medical Research Council Breathlessness scale (grade 2-5) [17]. Participants must be willing to be randomised to either arm of the study. They will be excluded if they have had an exacerbation in the last 4 weeks that required antibiotic or steroid therapy (including hospitalisations), recent or current pneumothorax or haemoptysis, and recent thoracic, gastric or facial surgery, or trauma. Participants must be able to communicate sufficiently well in English in order to provide informed consent.

The sample size was calculated based on a 0.5 point change in the Chronic Respiratory Questionnaire-Dyspnoea domain, with a standard deviation of 0.85 accounting for a type 1 error of 0.05 and $80 \%$ powered. This requires a sample size of 94 participants to complete the protocol $(\mathrm{n}=47$ per group). This was inflated by $13 \%$ as seen in the previous feasibility study to account for dropout; therefore, we anticipate recruiting 106 participants to the study [15]. The recruitment phase will run for a period of up to 3 years and will be terminated by December 2020 or once adequate power is achieved.

\section{Setting}

Participants in this study will be primarily recruited from research databases at the NIHR Leicester Biomedical Research Centre - Respiratory (Leicester, UK). Participants will also be identified by COPD clinics and Pulmonary Rehabilitation clinics at the University Hospital of Leicester NHS Trust. All potentially suitable participants will receive an invitation letter on behalf of the study team that includes the patient information sheet about the study. Potential participants will be given at least $24 \mathrm{~h}$ to review the information and consider their participation in the trial.

\section{Intervention}

This study will compare the Aerosure Medic (a HFAO device) with a sham control device.

The Aerosure Medic was developed by Actegy (Bracknell, UK). It is a battery-operated, dual-action device providing oscillations at either 15 or $25 \mathrm{~Hz}$ and flow resistance. The resistance applied ranges from 0 to $50 \mathrm{cmH}_{2} \mathrm{O}$ dependent on the patient's flow rate; however, the specific resistance cannot be set through this device. The device requires participants to inhale and exhale through a mouthpiece deeply for a minimum of $5 \mathrm{~min}$ at a time and repeat this three times per day. The oscillation will be performed on the $25 \mathrm{~Hz}$ setting. This is comparable to respiratory muscle training literature and is required for a minimum of 6 weeks in order to demonstrate effectiveness. As many of the outcome measures require a 2-week recall, the intervention phase was set at 8 weeks in order to capture changes that may have taken place. Participants will record each session in a self-reported diary and score each session on their perceived exertion based on a $0-10$ visual analogue scale. The intervention will be administered by a respiratory physiotherapist and the technique will be checked. Patients will be able to call the research department with any queries or problems with using the device.

The sham device appears identical to the active HFAO; however, the valve is removed and therefore provides no flow resistance or oscillations (figures 2 and 3).

Participants will receive the device intact, and will be instructed on its use and necessary cleaning requirements. Participants will be unaware of the device mechanics, and therefore will be unable to distinguish between the sham and active device. Participants and the outcome assessor will remain blinded to the treatment group. After the 8-week intervention phase each participant will be given the active HFAO device to retain.

Randomisation will be performed using a web-based programme and allocated on a 1:1 ratio (www. sealedenvelope.com). Randomisation will be allocated by an unblinded assessor and concealed from the patient and the outcome assessor.

\section{Outcomes}

The outcomes measures will be administered at baseline prior to commencement of the intervention phase. All outcome measures will be repeated 8 weeks later. Upon completion of the follow-up, all participants will be issued the HFAO device to use according to their own requirements. At 3 months after their final visit, participants will receive a postal follow-up that will consist of the self-reported Chronic Respiratory Questionnaire (CRQ-SR) and the COPD Assessment Test (CAT) alongside a form to collect average device use. Outcomes will explore lung function, health-related quality of life, exercise capacity and physical activity. 


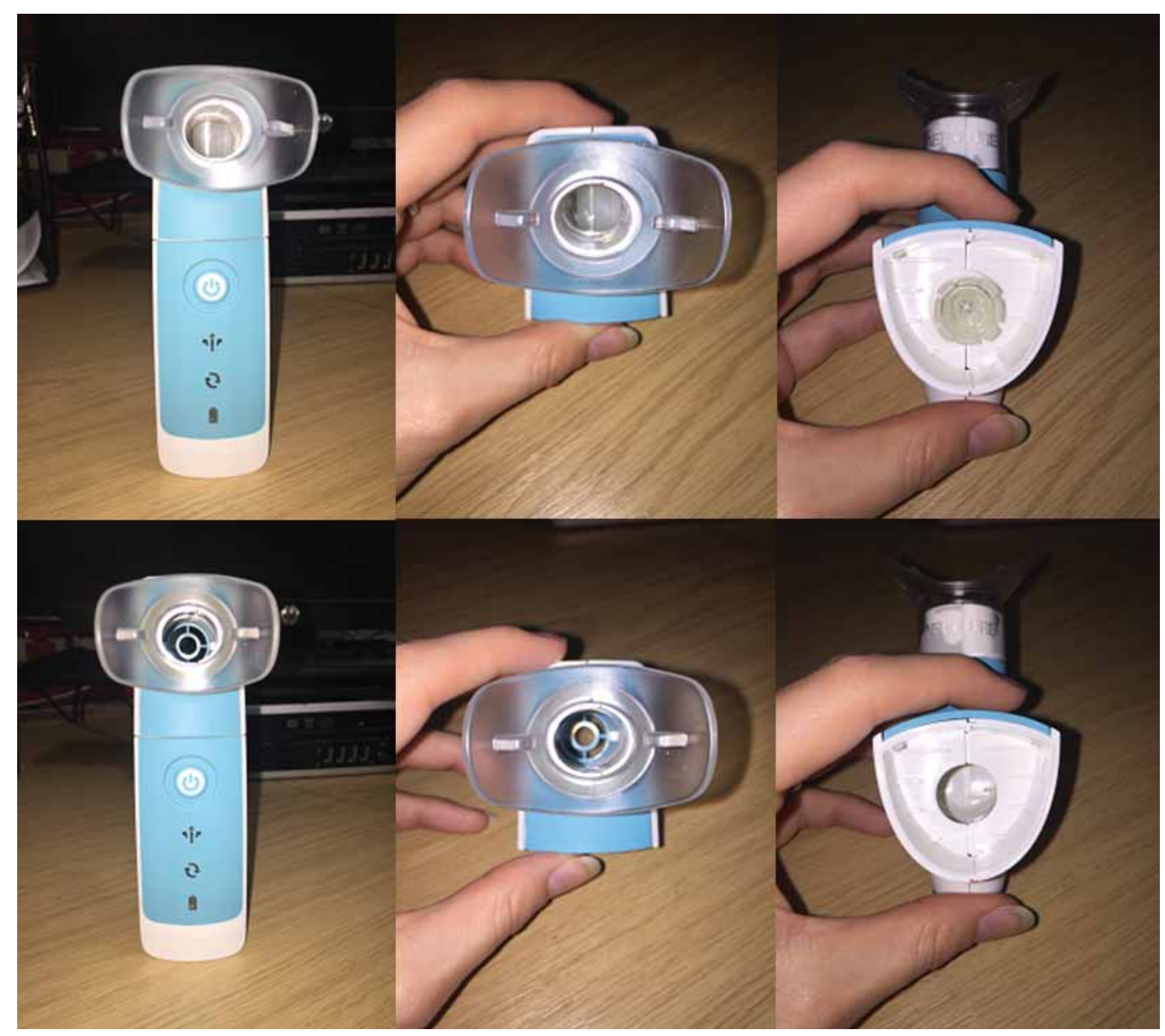

FIGURE 2 Top (left to right): Aerosure Medic active device, and device aerosol head anterior and inferior views. Bottom (left to right): Aerosure Medic sham device, and device aerosol head anterior and inferior views. Note mechanism removed from sham device.

\section{Primary outcome}

The primary outcome measure is the CRQ-SR dyspnoea domain administered at baseline, after the 8-week intervention phase and 3 months after the intervention phase [18]. The primary end-point is after the 8 -week intervention phase.

\section{Lung function}

Lung function will be assessed by spirometry (Vitalograph ALPHA, Maids Moreton, UK) to confirm diagnosis, performed in line with American Thoracic Society (ATS)/European Respiratory Society (ERS) standards [19]. While it is unlikely that the device will have an impact on spirometry, it will be repeated at follow-up in order to compare with other measurements. Spirometry is to be repeated a minimum of three times and up to seven times where the forced vital capacity falls outside of a $10 \%$ variance [16]. The best attempt will be recorded.

Respiratory muscle strength will be assessed on inspiration and expiration. Participants will perform this test from residual volume or full vital capacity for inspiratory and expiratory pressures, respectively. Patients will be instructed to inhale/exhale forcefully and sustain this for $2 \mathrm{~s}$. This is to be repeated a minimum of four times and up to seven attempts if participants continue to improve [20]. The measured best will be recorded. Participants will be considered to exhibit inspiratory muscle weakness if the results are $<60 \mathrm{cmH}_{2} \mathrm{O}$. Predicted values will be calculated for both inspiratory and expiratory pressures. Planned subgroup analysis will be performed on those who demonstrate inspiratory muscle weakness based on a cut-off of $60 \mathrm{cmH}_{2} \mathrm{O}$.

\section{Health-related quality of life}

Participants will complete questionnaires on their perceived health status and health-related quality of life. These will be completed independently with a researcher on hand to resolve any difficulties. The CRQ-SR, 


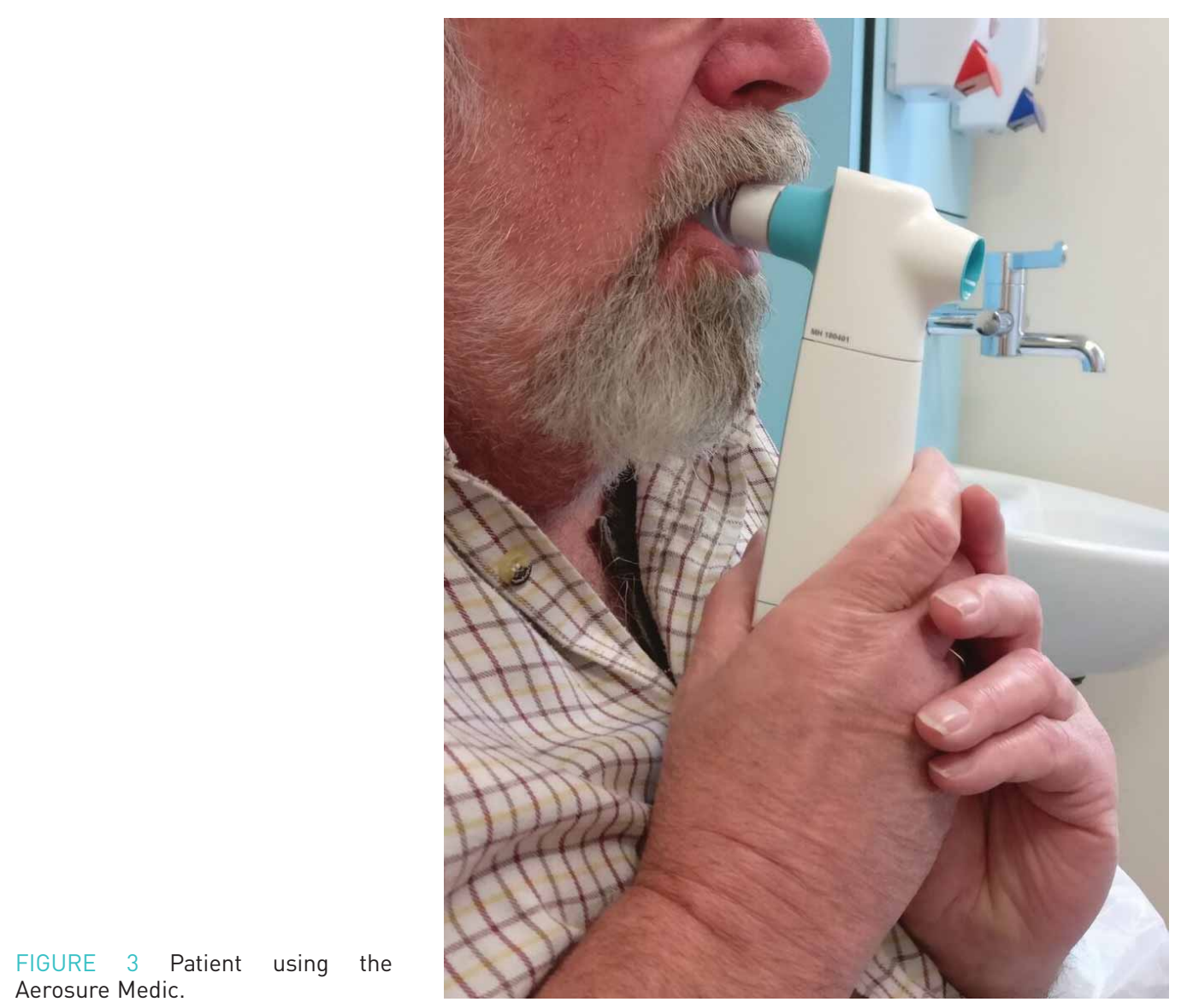

CAT, Leicester Cough Questionnaire, London Chest Activity of Daily Living questionnaire, Multidimensional Dyspnoea Profile, and Hospital Anxiety and Depression Scale will be used [18, 21-24]. All questionnaires will be scored individually and repeated at follow-up (8 weeks). The CRQ-SR and CAT will be repeated via a postal follow-up 3 months post-completion of the intervention phase. These two questionnaires and a survey asking about device use will be administered 3 months post-intervention phase to understand the long-term benefits of using the device. A subgroup analysis of "sputum producers" and "non-sputum producers" as stratified by the CAT sputum score will be explored.

\section{Exercise capacity}

Exercise capacity will be measured by the incremental shuttle walk test (ISWT) and the endurance shuttle walk test (ESWT) $[25,26]$. These are standardised and validated tests of maximal performance. Each participant will perform a familiarisation of the ISWT on visit 1 which is to be repeated after adequate rest, this will be at least $30 \mathrm{~min}$ and will require participant's baseline measurements to return to their pre-exercised state (blood pressure, oxygen saturations, heart rate and Borg breathlessness). The maximum distance performed on the ISWT will be used to calculate the appropriate speed for the ESWT, walking at $85 \%$ maximum. Both the ISWT and ESWT will be performed at baseline and follow-up post-intervention phase, and in line with ERS/ATS standards [27].

Physical activity

Physical activity is an exploratory outcome and participants will be required to wear an ActiGraph GT3X activity monitor (ActiGraph, Pensacola, FL, USA) for 1 week prior to randomisation and 1 week before visit 3 at the end of the intervention phase. The ActiGraph GT3X is to be worn around the waist, above the right hip, for 7 days and nights to the participant's tolerance. The ActiGraph GT3X is not to be worn during water-based activities. Data collected will be analysed using ActiLife software (ActiGraph).

\section{Ventilation heterogeneity}

Ventilation heterogeneity as measured via a multiple breath washout will be used as an exploratory outcome. This test is performed using an inert tracer gas (sulphur hexafluoride $\left(\mathrm{SF}_{6}\right)$ ) and measuring the 
washout phase. The patient is required to inhale and exhale through the mouthpiece with a nose clip in situ at tidal volumes. Initially patients will be breathing $\mathrm{SF}_{6}$ and then this will be disconnected once the desired concentration is achieved, leaving the patient to breathe room air through the mouthpiece until the $\mathrm{SF}_{6}$ gas is washed out to $1 / 40$ th of the starting concentration (figure 4). This is performed in triplicate and analysed for the mean of the three tests. Analysis is performed using a customised MATLAB algorithm. Each test must be within $10 \%$ functional residual capacity (FRC) to be included in the analysis. In the event one test is outside of this variance, the two remaining tests will be used [28, 29]. This analysis will produce the LCI, functional residual capacity (FRC), Scond and Sacin. LCI and FRC are calculated as the mean of the eligible tests, and Scond and Sacin will be calculated by a pooled slope analysis of the eligible tests using an automated MATLAB algorithm for phase 3 slope identification. This measure will be used to explore the impact of sputum clearance on ventilation heterogeneity and may provide clinical insight into the physiological implications of sputum retention. Each participant will perform this test at baseline and following the intervention phase.

\section{Compliance}

Compliance to the intervention will be analysed by a self-reported diary. Participants will complete the diary daily alongside the device use. This will record the duration and frequency of sessions alongside the perceived exertion and any other comments the participant may have. Participants completing $\geqslant 75 \%$ of the allocated treatments will be considered compliant [15]. A subgroup analysis will explore the impact of compliance on the results of the study.

\section{Qualitative focus groups}

Participants of the randomised control trial will be invited to participate in focus groups after the completion of the trial. This will provide insight into the experiences of using a device for the management of symptoms, which is an area of little research. The aim of the focus groups will be to provide an understanding of the use of the device for the management of COPD. This may also give insight into the compliance of the device during and after the trial. Both the intervention and the sham group will be invited to participate, as from the completion of the study all groups will be given an active device to retain. Those who express interest at the time of consent will be invited to participate. We will aim to conduct a minimum of two focus groups with around 5-10 participants per group. The topic of discussion has been developed with an experienced qualitative researcher and members of the Centre of

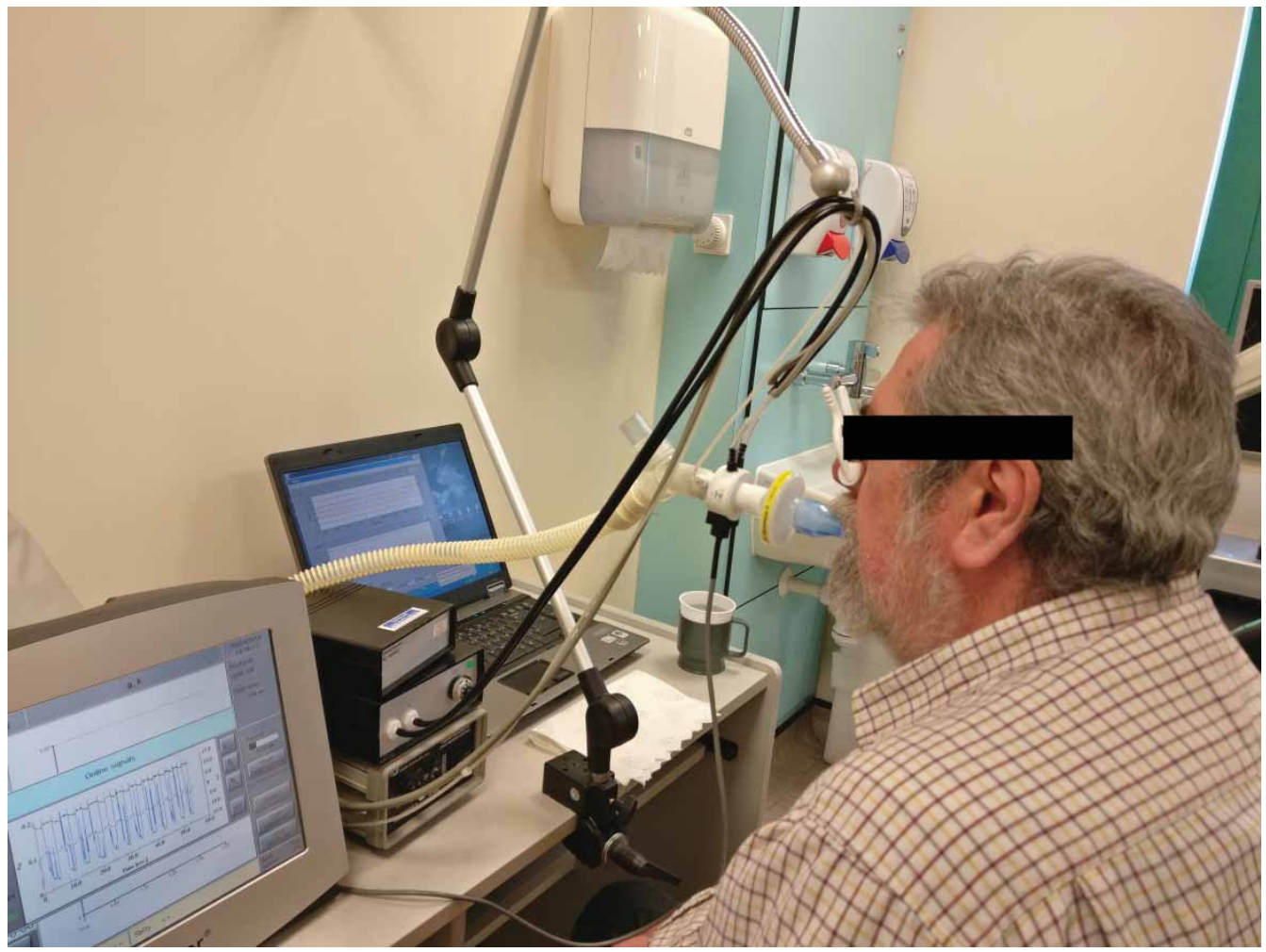

FIGURE 4 Patient performing a multiple breath washout measurement. 
Exercise and Rehabilitation Sciences Public and Patient Involvement advisory group. This will explore device use and impact on symptoms as well as patient preferences. The focus groups will be performed by a member of the team not directly involved with the randomised controlled trial, but who has experience in qualitative methods. The focus groups will be conducted in a hospital setting.

Data analysis plan

Baseline characteristics will be described and compared using the appropriate statistical tests (e.g. independent t-test, Mann-Whitney test or Chi-squared test). Changes pre- and post-intervention will be described for all of the aforementioned outcome measures, and also at 3 months for CRQ-SR and CAT. Differences between groups will be described, with the primary outcome being CRQ-SR at 8 weeks. Differences between groups will be compared using linear mixed models. Participants will need to complete the primary outcome measure after the intervention phase to be included in the analysis.

Pre-defined subgroup analyses will be performed on: 1) adherent versus non-adherent (defined as $<75$ or $\geqslant 75 \%$ self-reported use), 2) those with inspiratory muscle weakness $\left.\left(<60 \mathrm{cmH}_{2} \mathrm{O}\right), 3\right)$ "sputum producers" versus "non-sputum producers" (defined using the CAT subscale) and 4) those with ventilation heterogeneity (defined using the LCI). Further exploratory analyses will be performed on physical activity measures and multiple breath washout measures.

Qualitative focus groups will be recorded with consent and later transcribed. Transcriptions will be analysed using thematic analysis, whereby they will be coded for the development of the main themes [30]. Themes will be checked with the participants involved in the focus groups to ensure the discussion was accurately captured.

\section{Ethics and dissemination}

Ethical approval was gained on the June 7, 2017. The findings from this study will determine the clinical effectiveness of the HFAO device. Results will be disseminated widely to target key audiences, including healthcare professionals working in respiratory care, patients and members of the public, and academics and external organisations. We aim to disseminate nationally and internationally. The dissemination strategy will be developed alongside the Centre of Exercise and Rehabilitation Sciences Patient and Public Involvement advisory group (Glenfield Hospital, Leicester, UK). The results of this study will be presented at appropriate national, international and regional respiratory and physiotherapy conferences. Results will be published in a timely manner and are anticipated to be publically available during 2020 .

Acknowledgements: We would like to thank both the staff and members of the Centre of Exercise and Rehabilitation Sciences Patient and Public Involvement advisory group (Glenfield Hospital, Leicester, UK) who were involved with the development and conduct of this trial.

Author contributions: All authors co-developed the protocol. The manuscript was prepared by E. Daynes.

Conflict of interest: E. Daynes reports grants from Actegy Ltd during the conduct of the study. N. Greening has nothing to disclose. S. Sidiqqui has nothing to disclose. S. Singh reports grants from Actegy Ltd during the conduct of the study.

Support statement: This work was supported by Actegy Ltd (Bracknell, UK).

\section{References}

Laghi F, Tobin MJ. Disorders of the respiratory muscles. Am J Respir Crit Care Med 2003; 168: 10-48.

2 Gosselink R, De Vos J, van den Heuvel SP, et al. Impact of inspiratory muscle training in patients with COPD: what is the evidence? Eur Respir J 2011; 37: 416-425.

3 Beaumont M, Forget P, Couturaud F, et al. Effects of inspiratory muscle training in COPD patients: a systematic review and meta-analysis. Clin Respir J 2018; 12: 2178-2188.

4 Ottenheijm CA, Heunks LM, Dekhuijzen PN. Diaphragm muscle fiber dysfunction in chronic obstructive pulmonary disease: toward a pathophysiological concept. Am J Respir Crit Care Med 2007; 175: 1233-1240.

5 Evans J, Whitelaw W. The assessment of maximal respiratory mouth pressures in adults. Respir Care 2009; 54: $1348-1359$.

6 National Institute for Health Care Excellence. Chronic obstructive pulmonary disease in over 16s: diagnosis and management. 2010. www.nice.org.uk/guidance/cg101 Date last accessed: June 15, 2019.

7 Weiner P, Magadle R, Beckerman M, et al. Comparison of specific expiratory, inspiratory, and combined muscle training programs in COPD. Chest 2003; 124: 1357-1364.

8 ACCP/AACVPR Evidence-Based Guidelines Panel. Pulmonary Rehabilitation: joint ACCP/AACVPR evidencebased guidelines. Chest 2007; 131: 4S-42S.

9 Pauwels RA, Rabe KF. Burden and clinical features of chronic obstructive pulmonary disease (COPD). Lancet 2004; 364: 613-620.

10 Osadnik CR, McDonald CF, Jones AP, et al. Airway clearance techniques for chronic obstructive pulmonary disease. Cochrane Database Syst Rev 2012; 3: CD008328.

11 Davies J, Sheridan H, Bell N, et al. Assessment of clinical response to ivacaftor with lung clearance index in cystic fibrosis patients with a G551D-CFTR mutation and preserved spirometry: a randomised controlled trial. Lancet Respir Med 2013; 1: 630-638. 
12 Daynes E, Soares M, Greening N, et al. Repeatability of multiple breath washout measurements in stable Chronic Obstructive Pulmonary Disease. Eur Respir J 2018; 52: PA1075.

13 Daynes E, Soares M, Greening N, et al. The feasibility and repeatability of the lung clearance index via multiple breath washout measurements in stable chronic obstructive pulmonary disease. Thorax 2018; 73: A226-A227.

14 Bell AS, Lawrence PJ, Singh D, et al. Feasibility and challenges of using multiple breath washout in COPD. Int J Chron Obstruct Pulmon Dis 2018; 13: 2113-2119.

15 Daynes E, Greening NJ, Harvey-Dunstan TC, et al. High-frequency airway oscillating device for respiratory muscle training in subjects with COPD. Respir Care 2018; 63: 584-590.

16 Global Initiative for Chronic Obstructive Lung Disease. Global Initiative for Chronic Obstructive Lung Disease. Global Strategy for the Diagnosis, Management, and Prevention of Chronic Obstructive Pulmonary Disease. 2017. http://goldcopd.org/gold-2017-global-strategy-diagnosis-management-prevention-copd Date last accessed: June 15, 2019.

17 Stenton C. The MRC Breathlessness Scale. Occup Med 2008; 58: 226-227.

18 Williams JE, Singh SJ, Sewell L, et al. Development of a self-reported Chronic Respiratory Questionnaire (CRQ-SR). Thorax 2001; 56: 954-959.

19 Miller MR, Hankinson J, Brusasco V, et al. Standardisation of spirometry. Eur Respir J 2005; 26: 319-338.

20 American Thoracic Society/European Respiratory Society. ATS/ERS Statement on respiratory muscle testing. Am J Respir Crit Care Med 2002; 166: 518-624.

21 de Torres JP, Marin JM, Martinez-Gonzalez C, et al. Clinical application of the COPD Assessment Test: longitudinal data from the COPD History Assessment in Spain (CHAIN) cohort. Chest 2014; 146: 111-122.

22 Birring SS, Prudon B, Carr AJ, et al. Development of a symptom specific health status measure for patients with chronic cough: Leicester Cough Questionnaire (LCQ). Thorax 2003; 58: 339-343.

23 Garrod R, Bestall JC, Paul EA, et al. Development and validation of a standardized measure of activity of daily living in patients with severe COPD: the London Chest Activity of Daily Living scale (LCADL). Respir Med 2000; 94: 589-596.

24 Banzett RB, O’Donnell CR, Guilfoyle TE, et al. Multidimensional Dyspnea Profile: an instrument for clinical and laboratory research. Eur Respir J 2015; 45: 1681-1691.

25 Singh SJ, Morgan MD, Scott S, et al. Development of a shuttle walking test of disability in patients with chronic airways obstruction. Thorax 1992; 47: 1019-1024.

26 Revill SM. Further research on the Endurance Shuttle Walk Test (EWST) is needed before the test is advocated for clinical practice and research. Chron Respir Dis 2006; 3: 201.

27 Holland AE, Spruit MA, Troosters T, et al. An official European Respiratory Society/American Thoracic Society technical standard: field walking tests in chronic respiratory disease. Eur Respir J 2014; 44: 1428-1446.

28 Horsley A. Lung clearance index in the assessment of airways disease. Respir Med 2009; 103: 793-799.

29 Aurora P, Gustafsson P, Bush A, et al. Multiple breath inert gas washout as a measure of ventilation distribution in children with cystic fibrosis. Thorax 2004; 59: 1068-1073.

30 Braun V, Clarke V. Using thematic analysis in psychology. Qual Res Psychol 2006; 3: 77. 